\title{
THE CONSTRUCTION OF LOCI WITH A CYCLICAL SYMMETRY BY THE R-FUNCTIONS
}

\author{
YU.S. SEMERICH
}

A.N. Podgorniy's Institute for Problems in Mechanical Engineering of the National Academy of Sciences

Pozhgarskogo str. 2/10, 61046 Kharkov, Ukraine

E-mail: semerich@ipmach.kharkov.ua

Received October 11, 2004; revised January 23, 2005

\begin{abstract}
This paper deals with the development of the R-functions method for construction of equations describing a boundary of locus in the case of cyclical symmetry. The proposed method is based on transformations of coordinates and canonical locus.
\end{abstract}

Key words: R-functions method, locus, cyclical symmetry, transformation of coordinates

\section{Introduction}

A wide range of boundary value problems is connected to determination of physicalmechanical fields in domains possessing a translation symmetry. Usually solution of such problems is reduced to finding a field in the domain of translation element. However, in order to take a symmetry into account, we should require a symmetry of the field as well. Otherwise, the problem consists in finding a solution in the whole symmetrical domain. Application of the R-functions method for solution of such problems leads to the increased number of R-operations and basic domains, that are used to construct the symmetrical domain. As a result computation costs are increased essentially.

Thus it is important to develop a general modification of the R-functions method which can be used to describe the boundary of complex symmetrical locus. This methodology will increase the accuracy of approximations and will simplify the process of solution of boundary value problems by the R-functions method.

\section{The Function for Description of Symmetry}

First this problem was solved in the case of domains possessing a translation symmetry [1]. A canonical locus $\Sigma_{0}=\left(\sigma_{0}(x, y) \geq 0\right)$ and transformation of coordinates were used in this method. 
Let us consider a locus $\Omega=\bigcup_{i \in Z} \Sigma_{i}$, which consists of loci:

$$
\sigma_{i}(x, y)=\sigma_{0}(x-h i, y), \quad i \in Z,
$$

where $h$ is the step of translations. The loci $\Sigma_{i}, \Sigma_{i+1}, i \in Z$ can be separated by a periodical system of bands, which are perpendicular to the translation axis.

Let us construct a function $\mu_{n}(x, h)$ such that $\mu_{n}(x, h) \in C^{n}(R)$ and it is a linear function $(-1)^{k}(x-k h), k \in Z$ in $(k h-a, k h+a)$. Moreover, $\mu_{n}(x, h)$ is periodical with the basic period $2 h$. We define $\mu_{n}(x, h)$ in $[k h+a,(k+1) h-a]$, $k \in Z$ assuming that it is continuously differentiable and periodical. In addition we have that $\left|\mu_{n}(x, h)\right|>a$ for the $x \in[k h+a,(k+1) h-a], k \in Z$. Thus we obtain:

$$
\mu_{n}(x, h)=\frac{h}{\pi} \arcsin \left\{\frac{\sin \left(\frac{x \pi}{h}\right) C_{n}(a, h) S_{1}(a, h)}{S_{1}(a, h) C_{n}(a, h)+\left[1-S_{1}(a, h)\right] p^{n+1}(x, a, h)}\right\}
$$

here

$$
\begin{aligned}
& S_{1}(a, h)=\sin \left(\frac{\pi}{4}+\frac{a \pi}{2 h}\right), \quad S_{2}(x, a, h)=\sin ^{2}\left(\frac{x \pi}{h}\right)-\sin ^{2}\left(\frac{a \pi}{h}\right), \\
& p(x, a, h)=\left|S_{2}(x, a, h)\right|+S_{2}(x, a, h), \quad C_{n}(a, h)=2^{n+1} \cos ^{2(n+1)}\left(\frac{a \pi}{h}\right) .
\end{aligned}
$$

The plot of function (2.1) is presented in Fig. 1 for $h=2, a=0.5$.

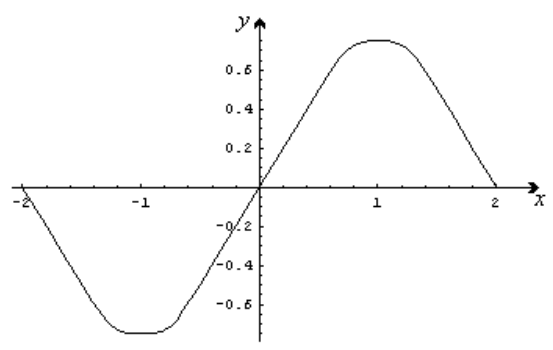

Figure 1. Function $\mu_{n}(x, 2), a=0.5$.

According to theorem [1], the boundary $\partial \Omega$ of locus $\Omega=\bigcup_{i \in Z} \Sigma_{i}$ s described by

$$
\omega(x, y) \equiv \sigma_{0}\left(\mu_{n}(x, h), y\right)=0
$$

\section{Cyclically Symmetrical Loci}

Let us construct the equation which describes the locus possessing a cyclical symmetry. In this paper we continue development of methods proposed in $[3,4,5]$. 
Suppose that $(\rho, \theta)$ are polar coordinates and $h=\frac{2 \pi}{m}$, here $m$ is a number of cyclically translated elements. Then function (2.1) can be written as:

$\mu_{n}(\theta, m)=\frac{2}{m} \arcsin \left\{\frac{\sin \left(\frac{m \theta}{2}\right) S_{1}(m, \alpha) C_{n}(m, \alpha)}{S_{1}(m, \alpha) C_{n}(m, \alpha)+\left[1-S_{1}(n, \alpha)\right] p^{n+1}(\theta, m, \alpha)}\right\}$,

here

$S_{1}(m, \alpha)=\sin \left(\frac{\pi+m \alpha}{4}\right), \quad S_{2}(\theta, m, \alpha)=\sin ^{2}\left(\frac{m \theta}{2}\right)-\sin ^{2}\left(\frac{m \alpha}{2}\right)$,
$C_{n}(m, \alpha)=2^{n+1} \cos ^{2(n+1)}\left(\frac{m \alpha}{2}\right), p(\theta, m, \alpha)=\left|S_{2}(\theta, m, \alpha)\right|+S_{2}(\theta, m, \alpha)$.

The plot of function (3.1) is shown in Fig. 2 for $m=3$.

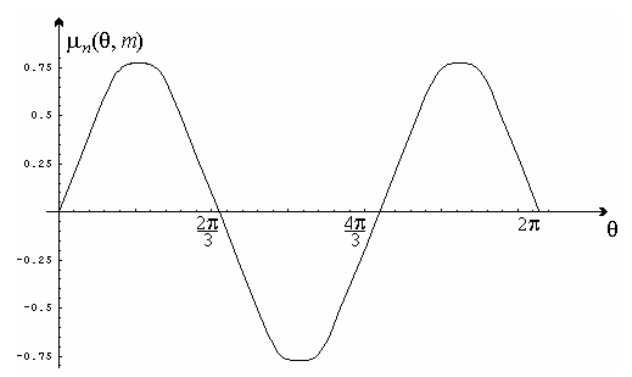

Figure 2. Function $\mu_{n}(\theta, 3)$.

Theorem 1. If the canonical locus

$$
\Sigma_{0}=\left[\sigma_{0}\left(x-r_{0}, y\right) \geq 0\right], \quad \sigma_{0}(x, y) \in C^{n}(\Omega),
$$

is symmetrical with respect to $O x$ axis and it can be placed inside the sector $-\alpha \leq$ $\theta \leq \alpha, 0<\alpha<\frac{\pi}{m}$, and all loci

$$
\Sigma_{i}=\left[\sigma_{0}\left(\rho \cos \left(\theta-\frac{2 \pi i}{m}\right)-r_{0}, \rho \sin \left(\theta-\frac{2 \pi i}{m}\right)\right) \geq 0\right]
$$

are received by turning the locus $\Sigma_{0}$ around the center of coordinates by angles $\frac{2 \pi i}{m}, i=0, \ldots, m-1$, then the equation, which describes the boundary $\partial \Omega$ of locus $\Omega=\bigcup_{i=1}^{m-1} \Sigma_{i}$ can be written as

$$
\omega(x, y) \equiv \sigma_{0}\left(\rho \cos \left(\mu_{n}(\theta, m)\right)-r_{0}, \rho \sin \left(\mu_{n}(\theta, m)\right)\right)=0 .
$$


Proof. Since function $\mu_{n}(\theta, m)$ is linear in intervals $\theta \in\left(\frac{2 \pi k}{m}-\alpha, \frac{2 \pi k}{m}+\alpha\right)$, $k=0, \ldots, m-1$, then equation (3.2) has the following form

$$
\omega(x, y) \equiv \sigma_{0}\left(\rho \cos \left((-1)^{k}\left(\theta-\frac{2 \pi k}{m}\right)\right)-r_{0}, \rho \sin \left((-1)^{k}\left(\theta-\frac{2 \pi k}{m}\right)\right)\right)=0
$$

Locus $\sigma_{0}(x, y) \in C^{n}(\Omega)$ is symmetrical with respect to $O x$ axis, thus this expression defines the boundary of domain $\Omega_{k}$, which is received by turning the locus $\Sigma_{0}$ by angle $\frac{2 \pi k}{m}, k=0, \ldots, m-1$. Since $\left|\mu_{n}(\theta, m)\right|>\alpha$ for $\theta \in$ $\left[\frac{2 \pi k}{m}+\alpha, \frac{2 \pi(k+1)}{m}-\alpha\right], k=0, \ldots, m-1$, then function (3.2) has no zero values. So, equation (3.2) is satisfied only on the boundary $\partial \Omega$ of locus $\Omega$.

Let us use Theorem 1 and construct the equation, defining the boundary of locus $\Omega$, which possesses a cyclical symmetry. As a canonical locus $\Sigma_{0}$ we choose the following locus

$$
\Sigma_{0}=\left[\sigma_{0}(x, y) \equiv \frac{1}{2 r_{1}}\left(r_{1}-\left(x-r_{0}\right)^{2}-y^{2}\right) \geq 0\right],
$$

which defines a circle of radius $r_{1}$ with the center in $\left(r_{0}, 0\right)$. Also we define elemental loci :

$$
\begin{aligned}
& \Sigma_{1}=\left[\sigma_{1}(x, y) \equiv \frac{1}{2 r_{2}}\left(r_{2}^{2}-x^{2}-y^{2}\right) \geq 0\right], \\
& \Sigma_{2}=\left[\sigma_{2}(x, y) \equiv \frac{1}{2 r_{3}}\left(r_{3}^{2}-x^{2}-y^{2}\right) \geq 0\right] .
\end{aligned}
$$

Locus $\Sigma_{1}$ defines a circle of radius $r_{2}$ with the center in $(0,0)$ and locus $\Sigma_{2}$ is a circle of radius $r_{3}$ with the center in $(0,0)$.

Then boundary equation of locus $\Omega$ possessing a cyclical symmetry can be written as:

$$
\omega(x, y) \equiv \overline{\sigma_{0}\left(\rho \cos \mu_{n}(\theta, m)-r_{0}, \rho \sin \mu_{n}(\theta, m)\right)} \wedge_{0} \overline{\sigma_{1}(x, y)} \wedge_{0} \sigma_{2}(x, y)=0 .
$$

Here we use $R$-operations with index $0:$ " $\wedge_{0}$ " is the $R$-conjunction and "-" is the $R$ negation. However, $R$-operations with indexes 1 or $\alpha$ are also used frequently [1].

Contour lines of function $\omega(x, y)$ for parameters $m=5,8$ are shown in Fig. 3.

Thus, the unique logical expression (3.5) and parameter $m$ allows us to construct various variants of equations, defining the boundary of cyclically symmetrical locus.

Let us consider a case, when locus $\Sigma_{0}$ is not symmetrical with respect to $O x$ axis. Then application of (3.2) to such a locus yields elements, which are mirror images of elements defined by (3.1) in intervals $\theta \in\left(\frac{2 \pi k}{m}-\alpha, \frac{2 \pi k}{m}+\alpha\right)$ for odd numbers $k$. In this case the following method can be used. 


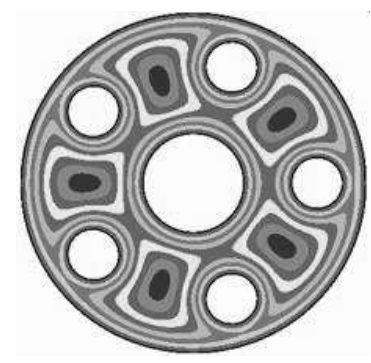

a) $m=5$

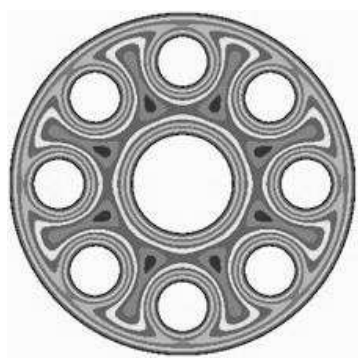

b) $m=8$

Figure 3. Contour lines of functions (3.5) for $r_{0}=5, r_{1}=1, r_{2}=2, r_{3}=7$.

Theorem 2. If canonical locus

$$
\Sigma_{0}=\left[\sigma_{0}\left(x-r_{0}, y\right) \geq 0\right], \quad \sigma_{0}(x, y) \in C^{n}(\Omega)
$$

is not symmetrical with respect to $O x$ axis and can be placed inside the following sector $-\alpha \leq \theta \leq \alpha, 0<\alpha<\frac{\pi}{m}$, and loci

$$
\Sigma_{i}=\left[\sigma_{0}\left(\rho \cos \left(\theta-\frac{2 \pi i}{m}\right)-r_{0}, \rho \sin \left(\theta-\frac{2 \pi i}{m}\right)\right) \geq 0\right], \quad i=0, \ldots, m-1
$$

are received by turning the locus $\Sigma_{0}$ around the center of coordinates by angles $\frac{2 \pi i}{m}$, $i=0, \ldots, m-1$, then the equation, defining the boundary $\partial \Omega$ of locus $\Omega=\bigcup_{i=1}^{m-1} \Sigma_{i}$ can be written as:

$$
\begin{aligned}
\omega(x, y) & \equiv\left\{\sigma_{0}\left(r \cos \mu_{n}(\theta, m)-r_{0}, r \sin \mu_{n}(\theta, m)\right) \wedge_{\alpha} \cos \left(\frac{m \theta}{2}\right)\right\} \\
& \vee_{\alpha}\left\{\sigma_{0}\left(r \cos \mu_{n}\left(\left(\theta-\frac{2 \pi}{m}\right), m\right)-r_{0}, r \sin \mu_{n}\left(\left(\theta-\frac{2 \pi}{m}\right), m\right)\right)\right. \\
& \left.\wedge_{\alpha} \cos \left(\frac{m}{2}\left(\theta-\frac{2 \pi}{m}\right)\right)\right\}=0 .
\end{aligned}
$$

Proof. The proof is based on results of Theorem 1. Note that the first term of (3.6) defines the boundary equation of cyclically translated locus without mirror elements, the second term defines the boundary equation of the same locus which is turned around by the angle $\frac{2 \pi}{m}$ and put into the place of its mirror image. The union of these loci defines the boundary equation of locus $\Omega$.

Let use Theorem 2 and construct boundary equation of locus $\Omega$, which is cyclically symmetrical. We define a canonical locus $\Sigma_{0}$ in the form of a predicate:

$$
\Sigma_{0}=\Sigma_{01} \bigcap \Sigma_{02},
$$

by using simple domains 


$$
\begin{aligned}
& \Sigma_{01}=\left[\sigma_{01}(x, y) \equiv(a-y) \geq 0\right], \\
& \Sigma_{02}=\left[\sigma_{02}(x, y) \equiv \frac{1}{2 r_{02}}\left(r_{02}^{2}-\left(x-r_{0}\right)^{2}-y^{2}\right) \geq 0\right] .
\end{aligned}
$$

Locus $\Sigma_{01}$ defines a half-plane below the line $y=a$ and locus $\Sigma_{02}$ defines a circle of radius $r_{02}$ with the center in $\left(r_{0}, 0\right)$. Also, we will use simple loci (3.3), (3.4), then boundary equation of locus $\Omega$ possessing cyclical symmetry can be written as:

$$
\begin{aligned}
\omega(x, y) & \equiv\left\{\overline{\left.\sigma_{0}\left(r \cos \mu_{n}(\theta, m)-r_{0}, r \sin \mu_{n}(\theta, m)\right) \wedge_{0} \cos \left(\frac{m \theta}{2}\right)\right\}}\right. \\
& \vee_{0}\left\{\overline{\sigma_{0}\left(r \cos \mu_{n}\left(\left(\theta-\frac{2 \pi}{m}\right), m\right)-r_{0}, r \sin \mu_{n}\left(\left(\theta-\frac{2 \pi}{m}\right), m\right)\right)}\right. \\
& \left.\wedge_{0} \cos \left(\frac{m}{2}\left(\theta-\frac{2 \pi}{m}\right)\right)\right\} \wedge_{0} \overline{\sigma_{1}(x, y)} \wedge_{0} \sigma_{2}(x, y)=0 .
\end{aligned}
$$

Contour lines of function $\omega(x, y)$ are shown in Fig. 4.

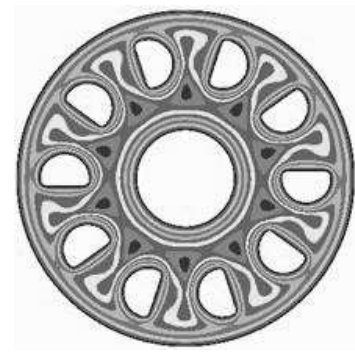

Figure 4. Contour lines of function (3.7) for $r_{0}=5, r_{02}=1, a=0.3, m=10$.

We note that application of the proposed method for the construction of boundary equations of cyclically symmetrical loci is not always possible. For example, such situation arises when two neighbour loci $\Sigma_{i}, \Sigma_{i+1}$ can not be separated from each other by sectors $-\alpha \leq \theta \leq \alpha, 0<\alpha<\frac{\pi}{m}$. Then the following method can be used.

Theorem 3. Let consider a canonical locus

$$
\Sigma_{0}=\left[\sigma_{0}\left(x-r_{0}, y\right) \geq 0\right], \quad \sigma_{0}(x, y) \in C^{n}(\Omega),
$$

which is not symmetrical with respect to $O x$ axis and can be placed inside the sector $-\alpha \leq \theta \leq \alpha, 0<\alpha<\frac{\pi}{m}$ and loci

$$
\Sigma_{i}=\left[\sigma_{0}\left(\rho \cos \left(\theta-\frac{2 \pi i}{m}\right)-r_{0}, \rho \sin \left(\theta-\frac{2 \pi i}{m}\right)\right) \geq 0\right], \quad i=0, \ldots, m-1,
$$

which are received by turning the locus $\Sigma_{0}$ around the center of coordinates by angles $\frac{2 \pi i}{m}, i=0, \ldots, m-1$. If loci $\Sigma_{i}, \Sigma_{i+1}$ can not be separated from each other 
by sectors $-\alpha \leq \theta \leq \alpha, 0<\alpha<\frac{\pi}{m}$, but loci $\Sigma_{i}, \Sigma_{i+2}$ can be separated, then the equation defining boundary $\partial \Omega$ of locus $\Omega=\bigcup_{i=1}^{m-1} \Sigma_{i}$ can be written as:

$$
\begin{aligned}
\omega(x, y) \equiv\left\{\left[\sigma_{0}\left(r \cos \mu_{n}(\theta, m)-r_{0}, r \sin \mu_{n}(\theta, m)\right) \wedge_{\alpha} \cos \left(\frac{m \theta}{4}\right)\right]\right. \\
\vee_{\alpha}\left[\sigma_{0}\left(r \cos \mu_{n}\left(\left(\theta-\frac{4 \pi}{m}\right), m\right)-r_{0}, r \sin \mu_{n}\left(\left(\theta-\frac{4 \pi}{m}\right), m\right)\right)\right. \\
\left.\wedge_{\alpha} \cos \left(\frac{m}{4}\left(\theta-\frac{4 \pi}{m}\right)\right)\right] \vee_{\alpha}\left[\sigma _ { 0 } \left(r \cos \mu_{n}\left(\left(\theta-\frac{2 \pi}{m}\right), m\right)-r_{0}\right.\right. \\
\left.\left.\quad r \sin \mu_{n}\left(\left(\theta-\frac{2 \pi}{m}\right), m\right)\right) \wedge_{\alpha} \cos \left(\frac{m}{4}\left(\theta-\frac{2 \pi}{m}\right)\right)\right] \\
\vee_{\alpha}\left[\sigma_{0}\left(r \cos \mu_{n}\left(\left(\theta-\frac{6 \pi}{m}\right), m\right)-r_{0}, r \sin \mu_{n}\left(\left(\theta-\frac{6 \pi}{m}\right), m\right)\right)\right. \\
\left.\left.\wedge_{\alpha} \cos \left(\frac{m}{4}\left(\theta-\frac{6 \pi}{m}\right)\right)\right]\right\}=0
\end{aligned}
$$

Remark 1. Function $\mu_{n}(\theta, m)$ in (3.8) was constructed assuming that $h=\frac{4 \pi}{m}$.

Proof. The proof is based on results of Theorem 1. Note that the first term in (3.8) defines boundary equation of cyclically translated locus whose elements don't contain mirror elements and are divisible by the angle $\frac{8 \pi}{m}$. The second term of (3.8) defines boundary equation of the same locus but turned around by the angle $\frac{4 \pi}{m}$. The third term defines the boundary equation of the first locus, which is turned around by the angle $\frac{2 \pi}{m}$. Elements of this locus have replaced mirror images, which are divisible by angle $\frac{2 \pi}{m}$. The last fourth term of (3.8) defines the boundary equation of the same first locus but turned around by the angle $\frac{6 \pi}{m}$. Now its elements have replaced mirror images, which are divisible by angle $\frac{4 \pi}{m}$. Thus the union of these loci defines the boundary equation of locus $\Omega$.

Theorem 3 can be used to construct the boundary equation of locus $\Omega$ possessing cyclical symmetry. Canonical locus $\Sigma_{0}$ is defined in the form of the following predicate:

$$
\Sigma_{0}=\left(\Sigma_{01} \bigcup \Sigma_{02}\right) \bigcap \overline{\Sigma_{03}}
$$

here we use simple loci

$$
\begin{aligned}
& \Sigma_{01}=\left[\sigma_{01}(x, y) \equiv \frac{1}{2 r_{01}}\left(r_{01}^{2}-\left(x-r_{0}\right)^{2}-y^{2}\right) \geq 0\right] \\
& \Sigma_{02}=\left[\sigma_{02}(x, y) \equiv \frac{1}{2 r_{02}}\left(r_{02}^{2}-\left(x-r_{0}\right)^{2}-\left(y-k_{1}\right)^{2}\right) \geq 0\right], \\
& \Sigma_{03}=\left[\sigma_{03}(x, y) \equiv \frac{1}{2 r_{03}}\left(r_{03}^{2}-\left(x-r_{0}\right)^{2}-\left(y+k_{2}\right)^{2}\right) \geq 0\right] .
\end{aligned}
$$


Locus $\Sigma_{01}$ defines a circle of radius $r_{01}$ with the center in $\left(r_{0}, 0\right)$, locus $\Sigma_{02}$ also defines a circle of radius $r_{02}$ with the center in $\left(r_{0}, k_{1}\right)$ and finally locus $\Sigma_{03}$ defines a circle of radius $r_{03}$ with the center in $\left(r_{0}, k_{2}\right)$. Also, we use simple loci (3.3), (3.4). By substituting the expression defining locus $\Sigma_{0}$ into (3.8), and applying $R$ operation " $\wedge_{0}$ " for loci $\overline{\sigma_{1}(x, y)}$ and $\sigma_{2}(x, y)$ we obtain the boundary equation of locus $\Omega$ possessing cyclical symmetry. The contour lines of this locus are shown in Fig. $5 a$.

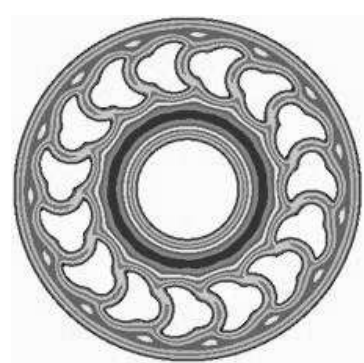

a)

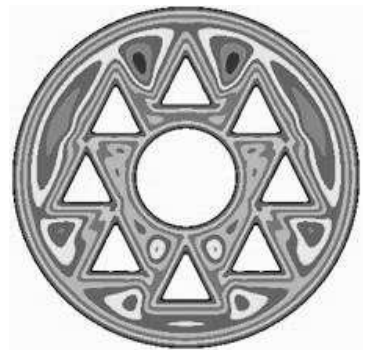

b)

Figure 5. Contour lines: a) function (3.8) for $r_{0}=5, r_{01}=1, r_{02}=0.5, r_{03}=1, k_{1}=1$, $k_{2}=-1, m=15$, b) function (3.9) .

If canonical locus $\Sigma_{0}$ is not symmetrical with respect to $O x$ axis, but a cyclical translation should preserve its orientation relatively to the Cartesian system of coordinates $x O y$ (see Fig. $5 b$ ), then the following method can be used.

Theorem 4. Let assume that a canonical locus

$$
\Sigma_{0}=\left[\sigma_{0}\left(x-r_{0}, y\right) \geq 0\right], \quad \sigma_{0}(x, y) \in C^{n}(\Omega)
$$

is not symmetrical with respect to $O x$ axis and it can be placed inside the sector $-\alpha \leq \theta \leq \alpha, 0<\alpha<\frac{\pi}{m}$, and loci

$$
\Sigma_{i}=\left[\sigma_{0}\left(\rho \cos \left(\theta-\frac{2 \pi i}{m}\right)-r_{0}, \rho \sin \left(\theta-\frac{2 \pi i}{m}\right)\right) \geq 0\right], \quad i=0, \ldots, m-1
$$

are received by turning the locus $\Sigma_{0}$ around the center of coordinates by angles $\frac{2 \pi i}{m}$, $i=0, \ldots, m-1$, preserving its orientation relatively to $x O y$ coordinate system, then the equation, which defines boundary $\partial \Omega$ of locus $\Omega=\bigcup_{i=1}^{m-1} \Sigma_{i}$ can be written as:

$$
\omega(x, y)=0
$$

where

$$
\omega(x, y) \equiv\left\{\sigma_{0}\left(x^{\prime}, y^{\prime}\right) \wedge_{\alpha} \cos \left(\frac{m \theta}{2}\right)\right\} \vee_{\alpha}\left\{\sigma_{0}\left(x^{\prime \prime}, y^{\prime \prime}\right) \wedge_{\alpha} \cos \left(\frac{m}{2}\left(\theta-\frac{2 \pi}{m}\right)\right)\right\},
$$




$$
\begin{gathered}
\left\{\begin{array}{l}
x^{\prime}=\left(r \cos \mu_{n}(\theta, m)-r_{0}\right) \cos \alpha_{k}-r \sin \mu_{n}(\theta, m) \sin \alpha_{k}, \\
y^{\prime}=\left(r \cos \mu_{n}(\theta, m)-r_{0}\right) \sin \alpha_{k}+r \sin \mu_{n}(\theta, m) \cos \alpha_{k},
\end{array}\right. \\
\left\{\begin{array}{l}
x^{\prime \prime}=\left(r \cos \mu_{n}\left(\left(\theta-\frac{2 \pi}{m}\right), m\right)-r_{0}\right) \cos \beta_{k}-r \sin \mu_{n}\left(\left(\theta-\frac{2 \pi}{m}\right), m\right) \sin \beta_{k} \\
y^{\prime \prime}=\left(r \cos \mu_{n}\left(\left(\theta-\frac{2 \pi}{m}\right), m\right)-r_{0}\right) \sin \beta_{k}+r \sin \mu_{n}\left(\left(\theta-\frac{2 \pi}{m}\right), m\right) \cos \beta_{k}
\end{array}\right. \\
\alpha_{k}=\theta-\mu_{n}(\theta, m), \quad \beta_{k}=\theta-\mu_{n}\left(\left(\theta-\frac{2 \pi}{m}\right), m\right) .
\end{gathered}
$$

A proof of this theorem is again based on results of Theorem 1. Let us note that coordinate transformation (3.10) keeps orientation of the canonical locus $\Sigma_{0}$ relatively to the Cartesian coordinate system $x O y$. We choose $\Sigma_{0}$ as a canonical locus, which defines a triangle, then contour lines of function $\omega(x, y)$, defined by (3.9), are shown in Fig. $5 b$.

In conclusion we note that contour plots were built by using system of engineering computations Polye-RL [2], which is developed at the department of Applied mathematics and computational methods of the Institute for Problems in Mechanical Engineering of the National Academy of Sciences, Ukraine.

\section{References}

[1] V.L. Rvachev. Theory of the R-functions and its some applications. Naukova dumka, Kiev, 1982. (in Russian)

[2] V.L. Rvachev and A.N. Shevchenko. Problem-oriented languages and systems for engineer computations. Tehnika, Kiev, 1988. (in Russian)

[3] Yu.S. Semerich. The R-functions method in the boundary value problem for a complex domain possessing the symmetry. Mathematical modelling and analysis, 8(1), 77 - 86, 2003.

[4] Yu.S. Semerich and T.I. Sheiko. Mathematical modeling methods of normalized equations for symmetrical loci. Bulletin Zaporozhye State University, 1, 71 - 84, 2003. (in Russian)

[5] A.V. Tolok, Yu.S. Semerich and T.I. Sheiko. Building symmetrical functions for symmetrical loci. Bulletin Zaporozhye State University, 2, 83 - 98, 2001. (in Russian) 


\section{Hodografo su cikline simetrija konstravimas $\mathbf{R}$ - funkciju metodu}

\section{Yu. Semerich}

Darbe pateiktas būdas, kaip pritaikyti R-funkcijų metodą taip vadinamų ciklinès simetrijos sričių kontūrų lygtims sudaryti. Pasiūlytasis metodas pagrịstas loordinačių transformavimu, pasinaudojant kanoniniu hodografu. 